\title{
Análisis de los mejores cursos de Ciencias Contables en São Paulo, según el examen de suficiencia CFC
}

\section{Analysis of the best accounting science courses in São Paulo, according to the CFC proficiency test}

\author{
Euclides Bezerra da Silva*1 \\ Pontificia Universidade Católica de São Paulo \\ euclidesbezerra@efficicont.com.br \\ Fernando De Almeida Santos ${ }^{1}$ \\ Pontificia Universidade Católica de São Paulo \\ almeidasantos@pucsp.br \\ Marcelo Eloy Fernandes ${ }^{2}$ \\ Fatec Barueri \\ marcelo.fernandes3@fatec.sp.gov.br \\ Windsor Espenser Veiga' \\ Pontificia Universidade Católica de São Paulo \\ windsor@pucsp.br
}

* Autor corresponsal.

1 Pontificia Universidade Católica de São Paulo (PUC-SP), Rua Ministro de Godoy, 969, São Paulo, BRASIL.

2 Fatec Barueri,

Av. Carlos Capriotti 123, Barueri, BRASIL.

\section{Resumen}

Propósito: La investigación consiste en un estudio comparativo de las cinco Instituciones de Educación Superior (IES) privadas del estado de São Paulo, donde aprueba más del 50\% de los candidatos en el examen de suficiencia del Consejo Federal de Contabilidad (CFC), señalando sus diferencias y similitudes.

Diseño/Metodología: Se utilizó una investigación exploratoria cualitativa, se separaron los temas y se clasificaron por eje temático y carga de trabajo, utilizando los lineamientos curriculares que incluyen formación básica, formación profesional, formación teórico-práctica y contenidos diferenciales con el fin de consolidar los resultados comparativos por eje temático de las instituciones encuestadas.

Resultados: Los resultados mostraron que las IES seleccionadas aplican, en sus contenidos de la carrera de Ciencias Contables, una carga de trabajo mayor al mínimo exigido por el Ministerio de Educación de 3000 horas, con variaciones entre el $8 \%$ y $47 \%$. Estas instituciones invierten en contenidos no obligatorios como sociología, psicología y otros.

Implicaciones: En cuanto al número de horas de formación básica, según la matriz curricular propuesta por el Consejo Federal de Contabilidad, cuatro de las cinco IES analizadas invierten entre un $13 \%$ y $48 \%$. Este hecho lleva a suponer que este conocimiento facilita una mejor comprensión en otras materias y proporciona una base sólida para la formación del alumno.

Originalidad/Valor: Hay cuatro de las cinco IES analizadas que invierten entre un $13 \%$ y un $48 \%$ por encima de la matriz curricular ajustada por la CFC en el grupo de formación básica, lo que facilita una mejor comprensión en otras materias y proporciona una base sólida para la formación del alumno. Limitaciones/Implicaciones: La investigación no abordó el contenido detallado cubierto por las Instituciones y las metodologías de enseñanza, porque no estaba disponible por las Instituciones.

Implicaciones prácticas: Este hecho demuestra la necesidad de revisar los contenidos de las instituciones en Brasil, para contribuir a la formación crítica de los profesionales.
INFORMACIÓN ARTÍCULO

Recibido: 25 de Abril 2021 Aceptado: 23 de Junio 2021

Palabras Claves:

Examen de suficiencia

Matriz curricular

Ciencias contables

Contabilidad 


\begin{abstract}
Purpose: The research consists of a comparative study of the five private Higher Education Institutions (IES), in the state of São Paulo, where more than $50 \%$ of candidates pass the proficiency exam of the Federal Accounting Council (CFC), pointing out their differences and similarities Design/Methodology: An exploratory, qualitative research was used, the topics were separated and classified by thematic axis and workload, using the curricular guidelines, which include basic training, professional training, theoretical-practical training and differential content with in order to consolidate the comparative results by thematic axis of the surveyed institutions.

Results: The results show that the selected HEIs, applied in their contents of the current science course, have a time load higher than the minimum required by the Ministry of Education of 3000 hours, with variations between $8 \%$ and $47 \%$. These institutions invest in non-mandatory contents such as sociology, psychology and among others.

Implications: Regarding the number of hours of basic training, according to the curricular matrix proposed by the Federal Accounting Council, four of the five HEls analyzed invest between $13 \%$ and $48 \%$. This fact leads to the assumption that this knowledge facilitates a better understanding of other contents; thus, providing a solid basis for student's training.

Original/value: There are four of the five HEls analyzed that invest between $13 \%$ and $48 \%$ above the curricular matrix adjusted by the CFC in the basic training group, which facilitates a better understanding of the other subjects and provides a solid foundation for student training.

Limitations/Implications: The research did not address the detailed content covered by the Institutions and teaching methodologies, as they were not available by the Institutions.

Practical implications: This fact demonstrates the need to review the course contents of institutions in Brazil to contribute to the critical training of professionals.
\end{abstract}

ARTICLE INFO

Received: 25 April 2021

Accepted: 23 June 2021
Keywords:

Proficiency test

Curriculum matrix

Accounting sciences

Accounting

\section{INTRODUCCIÓN}

Las entidades necesitan profesionales de contabilidad capacitados para satisfacer las demandas, porque son los responsables de generar información que respalde la toma de decisiones a medida que realizan trabajos de contabilidad, tales como: medición, registro, divulgación, gestión o análisis de demostraciones contables. En este contexto, el profesional necesita estar actualizado en derecho corporativo, derecho fiscal, derecho laboral, derecho fiscal, tecnología y sistemas de información, innovación, estrategia, marketing y otros. También en los procedimientos contables que la ley requiere, dependiendo de su área específica de especialización.

Para complementar el desarrollo social de un país que se basa en la educación, Silva y Rosa (2016) afirman que "[...] es a través de ella que un in- dividuo mejora su intelecto, su sabiduría, su conocimiento del mundo" (p.95). Cabe señalar que el mundo globalizado está en constante cambio, y ha ocasionado numerosas modificaciones y actualizaciones en todas las áreas, incluida la contabilidad y su desarrollo en la generación de información, a través de la educación.

Teniendo en cuenta diversos entendimientos, es relevante resaltar las actualizaciones legales constantes en el área de contabilidad, agregando un número complejo de Normas Contables Brasileñas (NBC) (CFC, 2011), alineadas con las Normas Internacionales de Contabilidad, conocidas como las International Financial Reporting Standards (IFRS).

Dados estos cambios, la presente investigación aborda los contenidos impartidos por las IES en el estado de São Paulo, con el fin de investigar la formación de licenciados en Contabilidad que se 
probaron a través del examen de suficiencia, aplicado por el Consejo Federal de Contabilidad (CFC) en 2017, específicamente en los dos exámenes de ese año.

El Examen de Suficiencia de CFC se introdujo en 1999 mediante la Resolución 853/99 de CFC, vigente hasta 2005, cuando fue terminado por orden judicial (Gonzales y Ricardino, 2017), con la alegación que no había ninguna ley que exigiera tal examen. En 2010, con la publicación de la Ley $N^{0} 12249$, que otorgó nuevos poderes al CFC, se revivió el examen de suficiencia, obligando al licenciado en contabilidad de Brasil a aprobarlo para tener el derecho registro como contador en el Consejo Regional de Contabilidad (CRC) de su estado.

Este hecho trajo un fortalecimiento de la profesión contable en el país, principalmente debido al crecimiento de la participación brasileña en los mercados internacionales y la evolución tecnológica de la información. También debe tenerse en cuenta que la profesión contable es la segunda categoría profesional que se regula mediante examen, antecedida por el Colegio de Abogados de Brasil (OBA) en 1996 (Girotto, 2012).

En el año 2011, el CFC comenzó la aplicación del examen de suficiencia en dos ediciones anuales, que hoy tiene un papel relevante para la sociedad porque contribuye a la evaluación y medición de la competencia en la formación del bachiller en Ciencias Contables. Por lo tanto, los contadores potenciales deben tener una licenciatura en contabilidad para poder tomar el examen de suficiencia y tener derecho a obtener el registro en los Consejos Regionales de Contabilidad (CRC) y actuar como contador en cualquier esfera de la sociedad.

Con el regreso de la aplicación del examen de suficiencia en 2011, por fuerza de ley de los dos exámenes en 2017, el CFC reveló los resultados del uso de las IES con relación a sus estudiantes.
Por consiguiente, esta investigación presenta un estudio exploratorio, cualitativo y comparativo, con las cinco IES privadas del estado de São Paulo que aprobaron más candidatos en el examen de suficiencia, utilizando exactamente los dos exámenes aplicados ese año por el CFC.

En tanto, gerentes y empresas buscan profesionales capacitados que puedan proporcionar herramientas e información para respaldar sus decisiones, por lo tanto, los profesionales que trabajan para ayudar en estas decisiones juegan un papel clave en la prosperidad del mundo empresarial. Para convertirse en contador es necesario estudiar la carrera de Contabilidad que pertenece al área de Ciencias Sociales aplicadas, con al menos cuatro años de estudios en IES. Por ley, la carrera debe ser aprobada en el examen de suficiencia aplicado por el CFC. Con respecto a ello, "El Consejo Federal de Contabilidad (CFC), siempre preocupado por garantizar un profesional de calidad a la sociedad, actuó en varios frentes implementando programas de inspección preventiva, educación continua y exámenes de certificación, incluido el examen de suficiencia" (Bugarim, Rodrigues, Pinho y Machado, 2014b, p.60)

Otra justificación para este estudio fue la observación de los datos consolidados en la Tabla 1, en referencia al período comprendido entre 2011 y 2017, y a la aplicación de 14 exámenes en los que participaron 477700 candidatos presentes en este período. En la evaluación aprobaron 171360 candidatos, equivalente al $35.87 \%$ y reprobó el 64.13\%, es decir, 306340 estudiantes o licenciados en Ciencias Contables no aptos para que actúen como contadores, prácticamente dos tercios de los inscritos en estos siete años. Es necesario establecer que, debido a los datos limitados de la investigación puede haber candidatos que hayan realizado el examen más de una vez para aprobar el período. La Tabla 1 muestra la tasa de fracaso:

Tabla 1. Consolidación de 14 pruebas de suficiencia de CFC 2011-2017.

Exámenes totales 14 ediciones de 2011 a 2017

\begin{tabular}{|c|c|c|c|c|}
\hline No. Candidatos Presentes & No. Aprobados & Aprobados & No. Rechazados & Rechazados \\
\hline 477700 & 171360 & $35.87 \%$ & 306340 & $64.13 \%$ \\
\hline
\end{tabular}


Cabe destacar en los indicadores del número de estudiantes matriculados en Brasil en la carrera de Educación Superior en Ciencias Contables, que esta representó el 6.4\% del total de matrículas de licenciatura (INEP, 2016). En Brasil en 2019, el Instituto Nacional de Estudios e Investigaciones Educativas Anísio Teixeira, dependiente del Ministerio de Educación, tenía 69781 matriculados, y en el mismo año había 10 455 egresados y se sabe que solo un tercio de este suele ser aprobado en suficiencia, no pudiendo ejercer la profesión posteriormente.

El problema de investigación consiste en: Las IES que obtuvieron los mejores indicadores en la aprobación del examen de suficiencia, ¿tienen una estructura curricular y una carga de trabajo que cumple con el plan de estudios propuesto por el CFC o tienen otros diferenciales que resalten esta aprobación?

El objetivo, por lo tanto, es realizar un estudio exploratorio comparativo y cualitativo con las instituciones privadas de educación superior del estado de São Paulo, mejor clasificadas en el examen de suficiencia con relación a la efectividad en aprobar este examen, identificando la matriz curricular y su carga, en comparación con la matriz curricular propuesta por la CFC.

\section{ESCENARIO DE LA CONTABILIDAD EN BRASIL}

La contabilidad en Brasil estuvo influenciada por una serie de líderes y culturas, la italiana, por ejemplo, cuna de la contabilidad que dio lugar al método de salidas plegadas, y los Estados Unidos con la entrada de industrias de ese país en la década de 1950, y una Contabilidad enfocada en prácticas y mecanismos de control para servir mejor a los usuarios. Esta visión de los estadounidenses se debe al colapso de la Bolsa de Nueva York en 1929 y al desarrollo del mercado de capitales que requirió nuevos estándares de contabilidad estandarizados, dando lugar a principios de contabilidad (CFC, 2016).

EI CFC afirma que, en 1974, la influencia de la escuela estadounidense comenzó a tomar forma y a ser parte de la investigación educativa con el desarrollo de nuevas teorías y prácticas contables, por lo que fue la base para la publicación de la ley principal que rige la Contabilidad en Brasil, Ley $\mathrm{N}^{\circ}$ 6404/76 (CFC, 2016).
Los cambios tecnológicos que impactan en varias áreas de la sociedad y otras relacionadas con la comprensión contable llevaron a la aparición de la Ley $N^{0} 11638 / 2007$, que modificó algunos aspectos de la legislación anterior y agregó regulaciones. De esta forma, la contabilidad brasileña comenzó importantes progresos y modificaciones, mejorando y expandiendo las relaciones comerciales en Brasil y en el extranjero, como también actualizaciones legales constantes, el avance de la tecnología, armonización contable, exámenes de evaluación, actualización educativa y el propio cambio de perfil del profesional contable.

La promulgación de la Ley N 11638/2007 y las modificaciones posteriores fueron factores esenciales para la armonización internacional de la contabilidad.

\section{RESULTADOS DEL EXAMEN DE SUFICIENCIA CFC}

El gobierno federal brasileño, mediante el Decreto Ley Nº 9295/46 creó el CFC, que actualmente prepara y aplica el Examen de Suficiencia. Como se describió, el profesional contable necesita tener un título en contabilidad y aun así ser aprobado. Este ha sido un desafío para muchos candidatos. Según una investigación realizada por Galvão (2016), utilizando cuestionarios para contadores registrados,

El $81.82 \%$ de los encuestados cree que el examen brinda reconocimiento profesional y el $82.73 \%$ se da cuenta de que el examen ayuda a seleccionar a los profesionales más capaces. El 89.55\% de los encuestados está a favor de la continuidad de la evaluación, el 53.61\% cree que esta evaluación necesita algunas mejoras (p. 49).

Desde la perspectiva de Miranda, Araújo y Miranda (2017), la investigación mostró una tasa de concordancia del $90 \%$ con el requerimiento de la prueba, aunque el $57.7 \%$ cree que la prueba debe tener revalidaciones periódicas, y el $84.6 \%$ entiende que además del examen, los profesionales deben demostrar una actualización continua. Es importante resaltar que, a pesar de estos aspectos que presenta el artículo, la obligación legal transforma la valoración necesaria para los profesionales contables. 
La Ley Nº 12249/2010, trajo la obligación para el curso de Bachillerato de Ciencias Contables, registrado en su artículo 12:

Los profesionales a los que se refiere este Decreto-Ley solo pueden ejercer la profesión después de la finalización regular de la Licenciatura en Ciencias en Contabilidad, reconocida por el Ministerio de Educación, la aprobación en el Examen de Suficiencia y el registro en el Consejo Regional de Contabilidad al que están sujetos. Ley $\mathrm{N}^{0}$ $12249 / 2010$.

Tabla 2. Resultados exitosos del examen: 2011 a 2017.

\begin{tabular}{|c|c|c|c|}
\hline Período de Examen & No. Candidatos Presentes & No. Aprobados & Aprobados Presentes \\
\hline $1^{\circ} / 2011$ & 13383 & 4130 & $30.86 \%$ \\
\hline $2^{\circ} / 2011$ & 18675 & 10886 & $58.29 \%$ \\
\hline $1^{\circ} / 2012$ & 24774 & 11705 & $47.25 \%$ \\
\hline $2^{\circ} / 2012$ & 29226 & 7613 & $26.05 \%$ \\
\hline $1^{\circ} / 2013$ & 33708 & 12000 & $35.60 \%$ \\
\hline $2^{\circ} / 2013$ & 36833 & 15891 & $43.14 \%$ \\
\hline $1^{\circ} / 2014$ & 38116 & 18824 & $49.39 \%$ \\
\hline $2^{\circ} / 2014$ & 32568 & 13591 & $41.73 \%$ \\
\hline $1^{\circ} / 2015$ & 38023 & 20715 & $54.48 \%$ \\
\hline $2^{\circ} / 2015$ & 38022 & 5580 & $14.68 \%$ \\
\hline $1^{\circ} / 2016$ & 41987 & 17576 & $41.86 \%$ \\
\hline $2^{\circ} / 2016$ & 40879 & 8948 & $21.89 \%$ \\
\hline $1^{\circ} / 2017$ & 46949 & 11860 & $25.26 \%$ \\
\hline $2^{\circ} / 2017$ & 44557 & 12041 & $27.02 \%$ \\
\hline
\end{tabular}

Fuente: CFC (2017).

La Tabla 2 muestra los resultados de los aprobados en el Examen de Suficiencia para el período 2011-2017:

La Tabla 2 muestra que desde el primer examen/2011 hasta el segundo examen/2017, el porcentaje más alto de aprobación fue en el segundo examen/2011 con un 58.29\%, equivalente a 10 886 aprobados, mientras que el último (considerado para esta investigación) presentó $27.02 \%$, es decir, 12041 aprobados.

Se nota que el número de candidatos inscritos/ presentes aumenta prácticamente con cada edición del examen, excepto en el período de crisis económica en el país que se extiende desde "el segundo trimestre de 2014, según el Comité de Datación de Ciclos Económicos (Codace) de la Fundación Getúlio Vargas [...] y el producto per cápita brasileño cayó un 9\% entre 2014 y 2016" (Barbosa Filho, 2017, p. 51). Esta es una de las principales razones por las que muchos estudiantes detuvieron sus estudios para priorizar otras áreas como la vivienda, la alimentación y la salud.
El examen de suficiencia presenta variables de porcentaje en los resultados de aprobación, y puede deberse a toda la complejidad de los últimos diez años, las adaptaciones y posiblemente las IES todavía se están ajustando a los cambios que ocurrieron en este período, lo que significa un gran desafío porque "las instituciones de educación contable tienen el desafío de proporcionar a sus estudiantes una educación de calidad que siga los cambios de la economía globalizada" (Bugarim, Rodrigues, Pinho y Machado, 2014a, p.122), además "las [...] instituciones de educación superior [...] tienen la misión de capacitar a profesionales calificados para un desempeño satisfactorio en la sociedad" (Bugarim y cols., 2014b, p.70).

El programa de estudios previsto en el Capítulo III, artículo 6 de la Resolución CFC No. 1486/2015 informa:

El Examen de Suficiencia consistirá en una prueba para los Licenciados en Ciencias Contables, sujeto a las siguientes condiciones y áreas de conocimiento: I. Contabilidad gene- 
ral; II Contabilidad de costos; III. Contabilidad Aplicada al Sector Público; IV. Contabilidad Gerencial; V. Contraloría; VI. Teoría contable; VII. Legislación profesional y ética; VIII Principios contables y normas contables brasileñas; IX. Auditoría contable; X. Experiencia en contabilidad; XI Nociones de derecho; XII Matemática Financiera y Estadística; XIII Lengua portuguesa. (CFC, 2015).

Dado lo anterior, se observa el perfil del profesional contable que, con el avance de la tecnología y el crecimiento en el flujo de información, obliga al contador a seguir una educación continua de manera constante, apuntando a los nuevos desafíos que plantea la profesión.

\section{Formación de profesionales contables en Brasil}

En Brasil, la formación del perfil del profesional contable vigente está contenida en la Resolución de la Junta Nacional de Educación de la Cámara de Educación Superior (CNE/CES) N 10, del 16 de Diciembre del 2004. Ella proporciona las pautas curriculares que deben adoptarse en las IES, y señala en su artículo 3 que el curso de Ciencias Contables debería empoderar al futuro contador en tres puntos fundamentales:

I. Comprender los problemas científicos, técnicos, sociales, económicos y financieros, a nivel nacional e internacional y en los diferentes modelos de organización; II. Tener dominio total de las responsabilidades funcionales que involucran investigaciones, auditorías, experiencia, arbitrajes, nociones de actividades actuariales y cuantificación de información financiera, patrimonial y gubernamental, con el uso completo de innovaciones tecnológicas; III. Revelar la capacidad crítico-analítica de evaluación, con respecto a las implicaciones organizacionales con el advenimiento de la tecnología de la información. (Resolución CNE/CES 10, 2004).

Además de lo expuesto, en su artículo 4 de la misma Resolución el profesional necesita tener las siguientes habilidades y destrezas:

I. Utilizar adecuadamente la terminología y el lenguaje de las Ciencias Contables y Actuariales; II. Demostrar una visión sistémica e interdisciplinaria de la actividad contable; III. Preparar opiniones e informes que contribuyan al desempeño eficiente y efectivo de sus usuarios, cualesquiera que sean los modelos orga- nizacionales; IV. Aplicar adecuadamente la legislación inherente a las funciones contables; V. Desarrollar con motivación y a través de una articulación permanente el liderazgo entre equipos multidisciplinarios, para capturar los insumos necesarios para los controles técnicos, la generación y difusión de información contable, con reconocido nivel de precisión; VI. Desempeñar sus responsabilidades con el dominio expresivo de las funciones contables, incluidas las nociones de actividades actuariales y la cuantificación de la información financiera, patrimonial y gubernamental, que permite a los agentes económicos y los gerentes de cualquier segmento productivo o institucional cumplir plenamente con sus deberes en lo que respecta a la gestión, los controles y la rendición de cuentas de su gestión ante la sociedad, generando también información para la toma de decisiones, la organización de actitudes y la construcción de valores orientados hacia la ciudadanía; VII. Desarrollar, analizar e implementar sistemas de información de control contable y de gestión, revelando la capacidad analítica crítica para evaluar las implicaciones organizacionales con la tecnología de la información; VIII. Ejercer con ética y competencia las atribuciones y prerrogativas que se le prescriben a través de la legislación específica, revelando dominios apropiados para los diferentes modelos organizacionales (Resolución CNE/CES 10, 2004).

Por su parte, Lemes y Miranda (2014) afirman que:

[...] es posible observar que hay flexibilidad y amplitud en las determinaciones de la Resolución CNE/CES No. 10/2004, que ayuda a cada institución de educación superior a definir qué competencias deben establecerse o reformularse en el escenario actual. Sin embargo, establece que el conocimiento, las habilidades y los métodos de enseñanza y aprendizaje deben estar en línea con las pautas establecidas del plan de estudios. Las directrices, a su vez, determinan la necesidad del desarrollo de habilidades relacionadas con la formación profesional, pero reafirman la necesidad de incorporar conocimientos organizativos, administrativos y de tecnología de la información; y desarrollar habilidades interpersonales, de comunicación y liderazgo... (p.301).

En complemento, Silva y Barbosa (2018) aclaran que "para que este proceso se lleve a cabo de ma- 
nera sólida, es necesario que el profesional en el área tenga una capacitación mínima que le permita realizar estas actividades, dada la necesidad del mercado" (p.115). La evolución actual, así como su desarrollo en el mundo, cuenta con elementos esenciales como la tecnología informática avanzada de alta tecnología, internet rápido y software que facilitan los procedimientos contables. Este conjunto de subsidios mejora los procesos repetitivos hasta el punto de despedir a un profesional contable.

Aun así, sobre la formación de profesionales de la contabilidad en Brasil, Tostes in Gonzales y Ricardino (2017), concluyen que "en el sistema brasileño, el conocimiento básico proporcionado en el curso de pregrado es aceptado como un requisito previo independientemente de la calidad variable de las universidades" (p.48). Es de destacar que el título de licenciado en Ciencias Contables se definió con una carga de trabajo mínima de 3000 horas y un límite mínimo de 4 años, previsto en la Resolución CNE/CES No. 2, 18 de Diciembre del 2007.

El Proyecto pedagógico del Curso (PPC), establecido en la Resolución CNE/CES N N 10, 16 de diciembre de 2004, en su artículo 2, determina que las IES establecen la organización curricular con la descripción de los siguientes aspectos:

I. Perfil profesional esperado para el estudiante, en términos de competencias y habilidades; II. Componentes integrales del plan de estudios; III. Sistemas de evaluación de estudiantes y cursos; IV. Pasantía supervisada; V. Actividades complementarias; VI. Monografía, proyecto de iniciación científica o proyecto de actividad, como el trabajo de conclusión del curso (TCC) como un componente opcional de la institución; VII. Régimen de oferta académica; VIII. Otros aspectos que hacen que este proyecto sea consistente (Resolución CNE/ CES 10, 2004).

Para cumplir con el PPC, las IES elaboran sus matrices curriculares y, de esta forma, cumplir fundamentalmente con el artículo 5 de la misma Resolución CNE/CES 10, 16 de diciembre de 2004, que establece:

Los cursos de graduación en contabilidad, licenciatura, deben incluir, en sus proyectos pedagógicos y en su organización curricular, contenidos que revelen conocimiento del escenario económico y financiero nacional e internacional, a fin de proporcionar la armonización de las normas y estándares inter- nacionales de contabilidad, de acuerdo con la capacitación requerida por la Organización Mundial del Comercio y las peculiaridades de las organizaciones gubernamentales, observando el perfil definido para el estudiante [...]. (Resolución CNE/CES 10, 2004).

En el mismo artículo 5, la Resolución CNE/CES No. 10, 16 de diciembre de 2004, complementa los contenidos interconectados de capacitación del contador, divididos en tres partes:

I. Contenidos de formación básica: estudios relacionados con otras áreas del conocimiento, especialmente Administración, Economía, Derecho, Métodos cuantitativos, Matemáticas y Estadística; II. Contenidos de formación profesional: estudios específicos relacionados con las teorías contables, incluidas las nociones de actividades actuariales y cuantificación de información financiera, patrimonial, gubernamental y no gubernamental, auditorías, experiencia, arbitraje y control, con sus aplicaciones propias de sector público y privado; III. Contenido de la capacitación teórico-práctica: prácticas supervisadas, actividades complementarias, estudios independientes, contenidos opcionales, práctica de laboratorio de computación utilizando software actualizado para contabilidad (CNE/CES Resolución 10, 16/12/2004).

La división de estos contenidos es la base para que las IES elaboren sus matrices curriculares, siendo de gran relevancia y desafío para las instituciones que necesitan adaptarse de acuerdo con la región, cultura y necesidades que demanda el mercado y la sociedad.

Es importante relevar que el plan de estudios tiene disciplinas de carácter de formación del ciudadano, como nociones de Derecho, Matemáticas, Ética y Lengua Portuguesa, con el objetivo de evaluar al individuo como un ser pensante. El examen de suficiencia debe estar estrechamente relacionado con la educación contable, por lo tanto, es bastante notable para la sociedad, sin embargo, es un tema muy poco explorado por los investigadores actuales.

La Tabla 3 presenta un resumen de las principales publicaciones sobre Educación Contable, capacitación de profesionales contables y el Examen de Suficiencia, de 2016 a 2020. 
Tabla 3. Resumen de las investigaciones realizadas sobre Educación Contable - 2016 a 2020.

\begin{tabular}{|c|c|}
\hline Fuente & Propósito de la Investigación \\
\hline Arantes y Silva (2020) & $\begin{array}{c}\text { Enfrentar el nivel cognitivo del Examen de Suficiencia con las habi- } \\
\text { lidades y competencias requeridas del contador sobre la base de los } \\
\text { objetivos educativos de la Taxonomía de Bloom. }\end{array}$ \\
\hline Silva y Barbosa (2018) & $\begin{array}{c}\text { Buscando comprender las perspectivas e impresiones de los alumnos } \\
\text { en el examen, sobre todo teniendo en cuenta que las últimas edicio- } \\
\text { nes han traído una alta tasa de reprobación. }\end{array}$ \\
\hline Gonzales y Ricardino (2017) & $\begin{array}{c}\text { Aborda una mayor transparencia en la difusión de los resultados del } \\
\text { examen de suficiencia de CFC a la sociedad. }\end{array}$ \\
\hline Silva, Miranda y Pereira (2017) & $\begin{array}{l}\text { Evaluar si las instituciones que ofrecen cursos de Contabilidad con la } \\
\text { propuesta curricular del Consejo Federal de Contabilidad (CFC), pre- } \\
\text { sentan calificaciones más altas en ENADE. }\end{array}$ \\
\hline Silva y Rosa (2016) & $\begin{array}{c}\text { Realizar una investigación descriptiva y exploratoria a través de los } \\
\text { datos recabados en el sitio del Instituto Nacional de Estudios e Inves- } \\
\text { tigación Educativa Anísio Teixeira (INEP). }\end{array}$ \\
\hline Galvão (2016) & $\begin{array}{l}\text { Verificar la percepción de los contadores con respecto a la finalización } \\
\text { del Examen de Suficiencia y si esto brindó la oportunidad para que los } \\
\text { contadores se destaquen, y sugerencias para mejorar la evaluación. }\end{array}$ \\
\hline Bline, Perreault y Zheng (2016) & $\begin{array}{c}\text { Analiza la estructura y las fases para aprobar el examen de CPA (Certi- } \\
\text { fied Public Accountant / Contador Público Certificado) en los Estados } \\
\text { Unidos. }\end{array}$ \\
\hline Miranda y cols. (2017) & $\begin{array}{c}\text { Identificar la percepción de profesores y profesionales sobre el exa- } \\
\text { men de suficiencia para la profesión contable, así como evaluar su } \\
\text { modelo actual. }\end{array}$ \\
\hline Souza, Cruz y Lyrio (2017) & $\begin{array}{l}\text { Analizar si existe asociación entre la tasa de aprobación en el examen } \\
\text { de suficiencia contable con el desempeño de los estudiantes y la } \\
\text { calidad de los cursos de educación superior en Brasil. }\end{array}$ \\
\hline Silva, Durigon, Mattiello da Silva y Santos (2020) & $\begin{array}{c}\text { Identificar las percepciones de los estudiantes sobre la realización del } \\
\text { examen de suficiencia. }\end{array}$ \\
\hline
\end{tabular}

Fuente: elaboración propia.

Silva y Barbosa (2018, p.115), realizó una encuesta a los estudiantes para conocer su percepción y perspectivas sobre la profesión contable debido a las altas tasas de reprobación en el examen CFC y aclara que "para que este proceso se lleve a cabo de manera sólida, es necesario que el profesional del área cuente con una formación mínima que le permita realizar estas actividades, satisfaciendo las necesidades del mercado."

Gonzales y Ricardino (2017), discuten en su artículo claramente la divulgación de datos del examen de aptitud por parte de la CFC y concluyen que, en el sistema brasileño los conocimientos básicos proporcionados en la carrera de pregrado se aceptan como prerrequisito, sin tomar en cuenta la calidad variable de las facultades. En tanto, Bline y cols. (2016), analizan que el procedimiento para realizar el CPA en los Estados Unidos tiene un examen que varía de uno a cien puntos por sección y requiere una aprobación mínima del 75\%.

Miranda y cols. (2017), a su vez, destacan que los profesores investigados entienden que los buenos resultados en estos exámenes son importantes para la imagen de las IES. Este hecho, sumado a la ya mencionada obligación de los profesionales de trabajar en el área, hace que este examen sea muy relevante. Los investigadores Arantes y Silva (2020), consideran que los resultados indican que el Examen de Suficiencia no está alineado con el perfil esperado del contador y que el CFC necesita evaluar si los licenciados en Contabilidad en Brasil son capaces de monitorear globalmente prácticas contables, es decir, si los futuros profesionales cuentan con las habilidades y competencias requeridas en el escenario nacional e internacional. 


\section{METODOLOGÍA}

Esta investigación es exploratoria, comparativa y cualitativa y utilizó los resultados de los exámenes de suficiencia de CFC, el cruce de su matriz curricular con los datos de las matrices curriculares de las IES, así como su carga de trabajo. El objeto de estudio pretende tabular los dos últimos resultados de las dos últimas ediciones del examen de suficiencia de 2017, analizando las IES privadas del estado de São Paulo que más aprobaron en el examen de suficiencia.

El trabajo se desarrolló utilizando datos secundarios, que según los estudios de Ferreira (2015), incluyen la información "disponible [...] públicamente en organizaciones internacionales, nacionales o locales $[. .$.$] que el autor puede usar en su$ investigación, pero no fueron recolectados espe- cíficamente para este propósito" (p.49).

La base de datos para elegir las IES se basó en los dos exámenes aplicados por el CFC en 2017 en todo Brasil, compuesta con los siguientes datos:

- $1 e r$ examen 2017 participaron 1524 IES; $y$

- 2do examen 2017 participaron 1582 IES.

En el primer examen de 2017, se seleccionaron 12 IES, clasificadas como privadas, tomando como criterio dos puntos simultáneos:

1. Número mínimo de estudiantes matriculados en el examen de 50 candidatos en la IES; y

2. Aprobación de al menos un $50 \%$ de los candidatos presentes.

Las IES clasificadas como privadas, foco de este trabajo, y que se comportaron con estos resultados, aparecen clasificados en la Tabla 4:

Tabla 4. Clasificación - Instituciones privadas de educación superior - 1er examen CFC de 2017.

\begin{tabular}{|c|c|c|c|c|c|}
\hline Posición & Instituciones de Educación Superior & Inscritos & Presentes & Aprobados & $\begin{array}{l}\text { Presentes } \\
\text { Aprobados }\end{array}$ \\
\hline $1^{0}$ & SP - Universidad Presbiteriana Mackenzie & 70 & 61 & 45 & $73.77 \%$ \\
\hline $2^{\circ}$ & RS - Universidad do Vale do Rio dos Sinos & 62 & 57 & 40 & $70.18 \%$ \\
\hline $3^{\circ}$ & SP - Centro Universitario Fecap & 59 & 52 & 36 & $69.23 \%$ \\
\hline $4^{0}$ & SP - Trevisan Escola Superior de Negocios & 84 & 77 & 53 & $68.83 \%$ \\
\hline $5^{0}$ & SP - Universidad São Judas Tadeu & 227 & 199 & 131 & $65.83 \%$ \\
\hline $6^{0}$ & $\begin{array}{c}\text { MG - Pontificia Universidad Catolica de } \\
\text { Minas Gerais }\end{array}$ & 71 & 49 & 31 & $63.27 \%$ \\
\hline $7^{0}$ & $\begin{array}{l}\text { SP - Pontificia Universidad Catolica de } \\
\text { Campinas }\end{array}$ & 81 & 73 & 46 & $63.01 \%$ \\
\hline $8^{\circ}$ & $\begin{array}{l}\text { RS - Universidad de Caxias do Sul - Caxias } \\
\text { do Sul }\end{array}$ & 66 & 62 & 35 & $56.45 \%$ \\
\hline $9^{\circ}$ & $\begin{array}{l}\text { SP - Pontificia Universidad Catolica de São } \\
\text { Paulo }\end{array}$ & 58 & 51 & 28 & $54.90 \%$ \\
\hline $10^{\circ}$ & $\begin{array}{c}\text { PR - Pontificia Universidad Catolica do } \\
\text { Paraná }\end{array}$ & 67 & 59 & 31 & $52.54 \%$ \\
\hline $11^{\circ}$ & DF - Universidad Catolica de Brasília & 68 & 53 & 27 & $50.94 \%$ \\
\hline $12^{\circ}$ & $\begin{array}{c}\text { MG - Pontificia Universidad Catolica de MG } \\
\text { - São Gabriel }\end{array}$ & 79 & 63 & 32 & $50.79 \%$ \\
\hline
\end{tabular}

Fuente: CFC (2017)

En el primer examen de 2017, como se muestra en la Tabla 4, se puede ver que los mejores logros que ocurrieron en la IES privadas se concentraron en la ciudad de São Paulo, específicamente en cinco instituciones educativas. Además, cabe destacar que las Pontificias Universidades Católicas aparecen en tres estados brasileños presentados en este ranking, a saber: Minas Cerais (dos instituciones), São Paulo (dos instituciones) y Paraná (una institución). 
Para el segundo examen de 2017, se aplicaron los mismos criterios utilizados para el primer examen, clasificados en privado y simultáneamente seleccionados de esta manera:

1. número mínimo de inscritos en el examen de 50 candidatos en la IES;

2. aprobación de al menos un 50\% de los candidatos.

Debido a que el estudio está dirigido a instituciones privadas de educación superior, el primer criterio que se verificará es la realización de los dos exámenes, sumando los candidatos presentes y determinando la media aritmética con relación a los aprobados.

La Tabla 5 muestra la concentración de aquellos aprobados eficientemente en la ciudad de São Paulo y también en las instituciones de las Pontificias Universidades Católicas. Por lo tanto, como se propuso al comienzo de la investigación, compararemos las matrices curriculares disponibles de las cinco instituciones en la ciudad de São Paulo para verificar si hay alguna similitud con la matriz de contenido programático propuesta por el CFC.

También participan en el Examen, egresados de instituciones públicas federales, pero utilizan una propuesta curricular presentada por la CFC (CFC, 2008), y existe una investigación sobre el tema realizada por Silva y cols. (2017), quienes detectaron que instituciones públicas obtuvieron las calificaciones más altas en ENADE, sugiriendo que las instituciones que tienen currículos similares al propuesto por la CFC presentan mejores resultados en ENADE. Se observa; sin embargo, que el perfil de las Universidades Públicas, por la gratuidad, es diferente, siendo más seleccionadas para la admisión y, ciertamente, impacta en los resultados finales. Por estas razones, la investigación se limitó a Universidades Privadas.

Tabla 5. Clasificación - IES privado - Participación promedio en los dos exámenes CFC 2017.

\begin{tabular}{ccccc}
\hline Posición & Instituciones de Educación Superior & Presentes & Aprobados & Media \\
\hline $1^{\circ}$ & SP - Centro Universitario Fecap & 172 & 123 & $71.51 \%$ \\
$2^{\circ}$ & SP - Trevisan Escola Superior de Negocios & 145 & 99 & $68.28 \%$ \\
$3^{\circ}$ & SP - Universidad São Judas Tadeu & 301 & 196 & $65.12 \%$ \\
$4^{\circ}$ & SP - Universidad Presbiteriana Mackenzie & 149 & 96 & $64.43 \%$ \\
$5^{\circ}$ & RS - Universidad do Vale do Rio dos Sinos & 111 & 67 & $60.36 \%$ \\
$6^{\circ}$ & MG - Pontificia Universidad Católica de Minas Cerais & 166 & 97 & $58.43 \%$ \\
$7^{\circ}$ & MG - Pontificia Universidad Católica de MG - São Cabriel & 122 & 69 & $56.56 \%$ \\
$8^{\circ}$ & PR - Pontificia Universidad Católica do Paraná & 129 & 72 & $55.81 \%$ \\
$9^{\circ}$ & SP - Pontificia Universidad Católica de São Paulo & 97 & 54 & $55.67 \%$ \\
\hline
\end{tabular}

Fuente: CFC (2017).

La metodología aplicada en este trabajo fue analizar las matrices curriculares de las cinco IES seleccionadas, buscando clasificar las disciplinas en cuatro ejes temáticos: Contenido por capacitación básica, Contenido de capacitación vocacional, Contenido de capacitación teórico-práctica y Contenido diferencial, según lo previsto en las pautas determinadas por la Resolución CNE/CES $N^{0} 10 / 2004$. Esta clasificación se realizó por adherencia según el modelo de matriz curricular propuesto por el CFC, junto con el modelo utilizado, por lo que se observa que en la clasificación existe un grado de subjetividad, por lo tanto, se utilizó 230 la clasificación más apropiada según la disciplina.

Silva y Rosa (2016), destacan que el objetivo es medir el desempeño de los estudiantes con relación al plan de estudios previsto en los lineamientos curriculares de la respectiva carrera de pregrado, y las habilidades y competencias en su formación, tanto para estudiantes de primer año como de último año. Por tal motivo, se utilizó para el análisis la mencionada Resolución.

Las IES enumeradas en la Tabla 5, seleccionadas por clasificación en los dos exámenes de Proficiencia en 2017, fueron: 
- FECAP - Centro Universitário Álvares Penteado (2018).

- TREVISAN - Trevisan Escola Superior de Negócios (2018).

- SÃo JUDAS - Universidade São Judas Tadeu (2018).

- MACKENZIE - Universidade Presbiteriana Mackenzie (2018).

- PUC-SP - Pontifícia Universidade Católica de São Paulo (2018).

Todos los datos secundarios se recopilaron en los sitios de las IES seleccionadas.

Las matrices curriculares se recopilaron en los sitios web de las respectivas IES, de acuerdo con la Ordenanza Normativa $N^{\circ} 40$ del 12 de diciembre del 2007, que instituyó que:

El e-MEC sistema de flujo de trabajo electrónico e-MEC y el sistema de gestión de la información para los procesos de regulación, evaluación y supervisión de la educación superior en el sistema educativo federal, y el registro e-MEC de instituciones y cursos superiores y consolida disposiciones sobre indicadores de calidad, banco de evaluadores (Base) y el Examen $\mathrm{Na}$ cional de Desempeño Estudiantil (ENADE) y otras disposiciones (Governo do Brasil, 2007).

La Ordenanza Normativa $N^{\circ} 40$, en el artículo 32, párrafo 1, punto IV, informa que:

Después de la autorización del curso, la institución se compromete a observar al menos el estándar de calidad y las condiciones bajo las cuales se otorgó la autorización, que se verificarán en el momento del reconocimiento y la renovación del reconocimiento. La institución debe publicar en un lugar visible con la secretaría de estudiantes, las condiciones de oferta del curso, indicando específicamente lo siguiente: matriz curricular del curso.

Asimismo, la Ordenanza Normativa No 40 refuerza el artículo 32, en el párrafo 2: "La institución mantendrá en su propio sitio web, y también en la biblioteca, para la consulta de estudiantes o partes interesadas, el registro oficial debidamente actualizado de la información a que se refiere el párrafo 1". Por lo tanto, los datos están disponibles en los sitios web de las IES seleccionadas y son públicos.

\section{RESULTADO DE LA INVESTIGACIÓN Y ANÁLISIS}

Utilizando la metodología presentada en el diagrama de flujo, fue posible llegar a los siguientes datos reflejados en la Tabla 6:

Los resultados presentados en la investigación mostraron que las instituciones educativas invierten más de 3000 horas de clases en la Licenciatura en Ciencias Contables. Este aumento en las horas de clase en las IES varió del 8\% al 47\%.

Las cuatro IES: FECAP, TREVISAN, MACKENZIE y PUC-SP invierten del $13 \%$ al $48 \%$ en el grupo de contenido de Capacitación Básica, por encima de la propuesta de matriz curricular de CFC.

Las IES primero forman al alumno para que sepa, reflexione y piense, proporcionando la base y la estructura para la formación inicial del alumno en la vida académica y una mayor facilidad de comprensión en las materias que tendrá que estudiar durante el curso.

Este punto puede justificar el resultado de porcentajes más altos de aprobación en el Examen de Proficiencia de CFC.

La IES SÃO JUDAS, fue la única que presentó una disminución del $11 \%$ en el grupo de contenido de capacitación básica en comparación con la matriz curricular propuesta por el CFC, pero invierte en otros grupos. Se cree que esta IES tiene otra filosofía de trabajo e inversión, que debería analizarse un poco más a fondo.

En cuanto al contenido de la formación profesional, las tres IES: TREVISAN, SÃO JUDAS y MACKENZIE, invirtieron entre $8 \%$ y $36 \%$ por encima de la propuesta curricular de CFC. MACKENZIE fue la que más invirtió (36\%). Mientras que FECAP y PUC-SP mostraron una disminución de $1 \%$ y $4 \%$ respectivamente en este contenido de enseñanza.

El contenido de capacitación teórico-práctica mostró que todas las IES se aplican por encima de la matriz curricular propuesta de CFC y el comportamiento fue: FECAP (33\%), TREVISAN (81\%), SÃO JUDAS (43\%), MACKENZIE (79\%) y PUC-SP (73\%). Este contenido es muy importante porque el alumno pone en práctica todo lo que aprendió en el aula. 
Tabla 6. Resumen consolidado comparativo de las cinco IES seleccionadas - 2017.

\begin{tabular}{|c|c|c|c|c|}
\hline Matriz curricular & Carga & Porcentaje & Propuesta & Variación \\
\hline FECAP & Horaria & En los grupos & CFC & $\mathrm{IES} \times \mathrm{CFC}$ \\
\hline Grupo - contenido de formación básico & 1200 & $33.33 \%$ & 810 & $148.15 \%$ \\
\hline Grupo - contenido de formación profesional & 1720 & $47.78 \%$ & 1740 & $98.85 \%$ \\
\hline Grupo - contenido de formación teórica práctica & 560 & $15.56 \%$ & 420 & $133.33 \%$ \\
\hline Grupo - contenido diferencial & 120 & $3.33 \%$ & 30 & $400.00 \%$ \\
\hline Total & 3600 & $100.00 \%$ & 3000 & $120.00 \%$ \\
\hline Matriz curricular & Carga & Porcentaje & Propuesta & Variación \\
\hline Trevisan & Horaria & En los grupos & CFC & IES $\times$ CFC \\
\hline Grupo - contenido de formación básico & 1040 & $27.37 \%$ & 810 & $128.40 \%$ \\
\hline Grupo - contenido de formación profesional & 1920 & $50.53 \%$ & 1740 & $110.34 \%$ \\
\hline Grupo - contenido de formación teórica práctica & 760 & $20.00 \%$ & 420 & $180.95 \%$ \\
\hline Grupo - contenido diferencial & 80 & $2.11 \%$ & 30 & $266.67 \%$ \\
\hline Total & 3800 & $100.00 \%$ & 3000 & $126.67 \%$ \\
\hline Matriz curricular & Carga & Porcentaje & Propuesta & Variación \\
\hline Universidad São judas & Horaria & En los grupos & CFC & IES $\times$ CFC \\
\hline Grupo - contenido de formación básico & 720 & $22.22 \%$ & 810 & $88.89 \%$ \\
\hline Grupo - contenido de formación profesional & 1880 & $58.02 \%$ & 1740 & $108.05 \%$ \\
\hline Grupo - contenido de formación teórica práctica & 600 & $18.52 \%$ & 420 & $142.86 \%$ \\
\hline Grupo - contenido diferencial & 40 & $1.23 \%$ & 30 & $133.33 \%$ \\
\hline Total & 3240 & $100.00 \%$ & 3000 & $108.00 \%$ \\
\hline Matriz curricular & Carga & Porcentaje & Propuesta & Variación \\
\hline Mackenzie & Horaria & En los grupos & CFC & IES $\times$ CFC \\
\hline Grupo - contenido de formación básico & 1164 & $26.29 \%$ & 810 & $143.70 \%$ \\
\hline Grupo - contenido de formación profesional & 2368 & $53.48 \%$ & 1740 & $136.09 \%$ \\
\hline Grupo - contenido de formación teórica práctica & 752 & $16.98 \%$ & 420 & $179.05 \%$ \\
\hline Grupo - contenido diferencial & 144 & $3.25 \%$ & 30 & $480.00 \%$ \\
\hline Total & 4428 & $100.00 \%$ & 3000 & $147.60 \%$ \\
\hline Matriz curricular & Carga & Porcentaje & Propuesta & Variación \\
\hline PUC - SP & Horaria & En los grupos & CFC & IES XCFC \\
\hline Grupo - contenido de formación básico & 918 & $26.36 \%$ & 810 & $113.33 \%$ \\
\hline Grupo - contenido de formación profesional & 1666 & $47.85 \%$ & 1740 & $95.75 \%$ \\
\hline Grupo - contenido de formación teórica práctica & 728 & $20.91 \%$ & 420 & $173.33 \%$ \\
\hline Grupo - contenido diferencial & 170 & $4.88 \%$ & 30 & $566.67 \%$ \\
\hline Total & 3482 & $100.00 \%$ & 3000 & $116.07 \%$ \\
\hline
\end{tabular}

Fuente: Datos compilados de la encuesta. IES x CFC matriz de currículo ajustada.

El contenido - diferenciales, demostró que todas Ias IES se aplican por encima del plan de estudios de CFC y presentó los siguientes indicadores: FECAP 120 horas de clases (300\%), TREVISAN 80 horas de clases (167\%), SÃO JUDAS 40 horas de clases (33\%), MACKENZIE 144 horas de clases (380\%) y PUC-SP 170 horas de clases (467\%). 


\section{CONSIDERACIONES}

Esta investigación, exploratoria y cualitativa, tuvo como objetivo analizar y comparar las matrices curriculares y la carga de trabajo aplicada en las cinco IES privadas del estado de São Paulo que más aprobaron el examen de suficiencia del CFC durante el año 2017, con relación a la matriz curricular ajustada del CFC.

En esta investigación se verificaron las similitudes y diferencias de las IES con la matriz curricular de CFC ajustada. Además de comparar con los cuatro grupos de contenido temático. El presente estudio también tuvo limitaciones de investigación con respecto a los datos recopilados en el sitio.

Los resultados obtenidos se lograron y mostraron factores que nos llevan a una reflexión. En forma aislada, FECAP fue el que más invirtió en contenido de capacitación básica, 48\% por encima del plan de estudios y fue el que más aprobó el examen de suficiencia (71.51\%).

Se observa que, en Brasil, las Instituciones Públicas utilizan la matriz curricular propuesta por el CFC y tienen una audiencia muy diferente a la de las instituciones privadas. Los públicos son, en su mayor parte, gratuitos y tienen una gran competencia para la admisión. Los privados tienen un mayor número de estudiantes y un proceso de selección de acceso más fácil para la admisión, por lo que la aprobación significa una mayor necesidad de desarrollo del estudiante.

Se cree que invertir más en educación básica, como matemáticas, portugués, economía, derecho, entre otros, facilitaría y capacitaría al alumno para que comprendiese todo el curso en su formación profesional. Otro factor fue que las IES investigadas mantienen en su matriz curricular, contenidos diferenciales como psicología y sociología, porque dan una mejor orientación al alumno de la sociedad que forma parte.

La investigación presentó entre las IES estudiadas que la estructura curricular, similar a la propuesta curricular de CFC, proporcionó más del $50 \%$ de la aprobación de los estudiantes. Este hecho deja discusiones abiertas y reflexiones que pueden mejorar la enseñanza de la contabilidad en las IES, si se realizan estos ajustes. Este estudio se puede utilizar como benchmarking en sus matrices curriculares.
La presente investigación puede conducir a una mayor investigación al profundizar en el tema, como los ejes aplicados en el contenido del examen de suficiencia, el comportamiento del estudiante y la clase social, ya sea trabajando o simplemente estudiando. También puede surgir investigación por país región x CFC, o medir si la calidad del profesor, su título y la actualización reflejan la mayor aprobación del examen.

Se espera que este trabajo pueda dar lugar a nuevos estudios centrados en la educación contable, como nuevos métodos de aprendizaje, llamados metodologías activas e investigación centrada en el examen CFC y ENADE, para obtener la mejor calidad en la formación profesional en contabilidad.

Esta investigación puede tener otros desarrollos que busquen analizar cuestiones relacionadas con el plan de estudios, en comparación con los nuevos exámenes de suficiencia. Otros temas también se pueden derivar de la evaluación del comportamiento de los estudiantes y de la clase social, buscando comprender las calificaciones del profesorado, así como nuevos métodos de aprendizaje colaborativo, involucrando metodologías activas, por ejemplo.

Finalmente, otros investigadores podrán evaluar investigaciones encaminadas a examinar la CFC y ENADE, a favor de una mejor calidad en la formación del profesional contable.

Las limitaciones del artículo, por otro lado, están asociadas a los datos recogidos en la página web de las IES, porque no se indica el nombre de la disciplina, lo que dificulta la selección del grupo específico, teniendo el investigador un criterio subjetivo en su clasificación.

\section{REFERENCIAS}

Arantes, D. A., y da Silva, D. M. (2020). Análise do nivel cognitivo do exame de suficiência contábil na perspectiva da taxonomia de bloom. Contabilidade Vista y Revista, 31(2).

Barbosa Filho F. H. (2017). A crise econômica de 2014/2017. Estudos Avançados, 31(89), 51-60.

Bline, D., S. Perreault, S. \& Zheng, X. (2016). An exploratory examination of order effects on CPA exam passage timelines. Journal of Accounting Education, 36, 65-74. 
Bugarim, M., Rodrigues, L., Pinho, J., y Machado, D. (2014a). Análise histórica dos resultados do Exame de Suficiência do Conselho Federal de Contabilidade. Revista Contabilidade e Controladoria, 6(1).

Bugarim, M. C. C., Rodrigues, L. L., Pinho, J. C. da C., y Machado, D. de Q. (2014b). O desempenho dos profissionais de contabilidade no exame de suficiência do CFC: uma análise de conglomerados regionais. Revista de Contabilidade e Organizações, 8(22), 60-71.

Centro Universitário Álvares Penteado (2018). Recuperado el 16 de Noviembre de 2018 de: https://www.fecap.br/graduacao/matriz-curricular/ciencias-contabeis.php.

Conselho Federal de Contabilidade (2008). Proposta Nacional de conteúdo para o curso de graduação em Ciências Contábeis. Acceso en 15 Dez, 2018. http://portalcfc.org.br/wordpress/wpcontent/uploads/2013/01/proposta.pdf.

Conselho Federal de Contabilidade (2011). Resolução 1328/2011. Estrutura das Normas Brasileiras de Contabilidade. Acceso en 10 Nov. 2017. , http://www1.cfc.org.br/sisweb/sre/ detalhes_sre.aspx?Codigo=2011/001328.

Conselho Federal de Contabilidade (2015). Resolução 1486/2015. Regulamenta o Exame de Suficiência como requisito para obtenção de Registro Profissional em Conselho Regional de Contabilidade (CRC). Acceso en 14 Dez 2017. http://cfc.org.br/registro/legislacao/.

Conselho Federal de Contabilidade (2016). 70 Anos de Contabilidade. Brasília: CFC.

Conselho Federal de Contabilidade (2017). Exame de Suficiência: CFC. Acceso 05 Out. 2019. https://cfc.org.br/exame-de-suficiencia-anteriores/10-exame-de-suficiencia-de-2017/

Decreto - Lei N 9295/46, de 27 de maio de 1946. Acceso 05 Out. 2019. http://www.planalto. gov.br/ccivil_03/decreto-lei/del9295.htm

Ferreira, M. P. (2015). Pesquisa em Administração e Ciências Sociais Aplicadas: um guia para Publicação de Artigos Acadêmicos. Rio de Janeiro: Editora LTC.

Galvão, N. (2016). Percepção dos Contadores sobre o Exame de Suficiência do CFC. Revista Catarinense da Ciência Contábil, 15(45), 49-62.
Girotto, M. (2012). Exame de Suficiência é instituído por lei. Revista Brasileira de Contabilidade, 184, 6-15.

Goberno do Brasil (2007). Ministerio da Educação. Enade. Acceso en 11 Mar. 2018. http://portal. mec.gov.br/enade.

Gonzales A. y Ricardino F. A. A. (2017). Transparência na divulgação de resultados do exame de suficiência do CFC. Revista Contabilidade Vista e Revista, 1(28), 45-66.

Instituto Nacional de Estudos e Pesquisas Educacionais Anísio Teixeira. Sinopse Estatística da Educação Superior (2016). Brasília: INEP, 2017. Recuperado de: http://portal.inep.gov.br/basica-censo-escolar-sinopse-sinopse el 18 de Junio de 2021.

Lei $N^{0}$ 6404/76, de 15 de dezembro de 1976. Acceso 15 octubre 2017. http://www.planalto.gov. br/ccivil_03/leis/L6404compilada.htm.

Lei $N^{0} 11638 / 07$, de 28 de dezembro de 2007. Acceso en 15 octubre 2017. http://www. planalto. gov.br/ccivil_03/_ato2007-2010/2007/lei/ l11638.htm.

Lei $\mathrm{N}^{0} 12249$, de junho de 2010 . Acceso en 15 octubre 2017. http://www.planalto.gov.br/ccivil_03/_ato2007-2010/2010/lei//12249.htm.

Lemes, F. D. y Miranda, G, J. (2014). Habilidades Profissionais Do Contador Preconizadas Pela Ifac: Um Estudo Com Profissionais Da Região Do Triângulo Mineiro. Advances in Scientific and Applied Accounting, 7(2), 293-316.

Miranda, C. S., Araújo, A. M. P., y Miranda, R. A. M. (2017). 0 exame de suficiência em contabilidade: uma avaliação sob a perspectiva dos pesquisadores. Revista Ambiente Contábil, 9(2), 158-178.

Pontifícia Universidade Católica de São Paulo PUC-SP (2018). Recuperado el 16 de Noviembre de 2018 de: https://www.pucsp.br/graduacao/ciencias-contabeis\#matriz-curricular.

Portaria Normativa No 40. 14 de dezembro de 2007. Acceso en 6 Marzo 2019. http://portal. mec.gov.br/index.php?option=com_docmany view=downloady alias= 16763-port-norm040-2007-seresy Itemid=30192.

Resolução CNE/CES 10 de 16/dez/2004. Acceso el 14 diciembre 2018. http://portal.mec.gov.br/ cne/arquivos/pdf/rces10_04.pdf. 
Resolução CNE/CES 02 de 18/jun/2007. Acceso el 14 De diciembre 2018. http://portal.mec.gov. br/cne/arquivos/pdf/2007/rces002_07.pdf.

Silva, C. M., y Barbosa, C. A. M. (2018). Perspectivas e Impressões Sobre o Exame de Suficiência do CFC na Visão de Discentes do Curso de Graduação de Ciências Contábeis. Revista de Auditoria Governança e Contabilidade, 6(222), 114-128.

Silva, J. V., Durigon, A. R., Mattiello da Silva, J. V. V., y Santos, R. (2020). O Exame de Suficiência na percepção dos alunos de Ciências Contábeis. Revista Catarinense Da Ciência Contábil, 19, 1-16.

Silva, G. M. y Rosa, F. S. (2016). O Curso de Ciências Contábeis no Brasil: um estudo sobre as políticas públicas de ensino superior e seu reflexo na oferta e na demanda no período de 2001 a 2013. Revista de Gestão, Finanças e Contabilidade, 6(2), 94-111.

Silva, V. R., Miranda, G. J. y Pereira, J. M. (2017). ENADE e Proposta Curricular do CFC: um Estudo em Cursos Brasileiros de Ciências Contábeis. Revista de Educação e Pesquisa em Contabilidade, 11(3), 261-275.
Souza, P. V. S., Cruz, U. L., y Lyrio, E. F. (2017). A relação do exame de suficiência contábil com o desempenho discente e a qualidade dos cursos superiores em Ciências Contábeis do Brasil. Revista Ambiente Contábil, 9(2), 179-199.

Trevisan (2018). Recuperado el 16 de Noviembre de 2018 de: https://www.trevisan.edu.br/ sao-paulo/graduacao/ciencias-contabeis-para-profissionais-graduados.

Universidade Presbiteriana Mackenzie (2018). Recuperado el 16 de Noviembre de 2018 de: https://www.mackenzie.br/graduacao/ sao-paulohigienopolis/ciencias-contabeis/ matriz-curricular/.

Universidade São Judas Tadeu (2018). Recuperado el 16 de Noviembre de 2018 de: https://www.usjt.br/app/uploads/2017/09/ Ci\%C3\%AAncias-Cont\%C3\%A1beis1.pdf. 
Revista Academia \& Negocios Vol.7 (2) 2021 pp. 221 - 236 